\title{
Computational Analysis of Alzheimer Amyloid Plaque Composition in 2D- and elastically reconstructed 3D-MALDI MS Images
}

Thomas Enzlein ${ }^{1,2,3}$, Jonas Cordes 4 , Bogdan Munteanu ${ }^{1}$, Wojciech Michno ${ }^{5,6}$, Lutgarde Serneels ${ }^{2,3}$, Bart De Strooper ${ }^{2,3,7}$, Jörg Hanrieder ${ }^{5,6,8}$, Ivo Wolf ${ }^{4}$, Lucía Chávez-Gutiérrez ${ }^{2,3}$, Carsten Hopf ${ }^{1, *}$

1. Center for Mass Spectrometry and Optical Spectroscopy (CeMOS), Mannheim University of Applied Sciences, Paul-Wittsack Str. 10, 68163, Mannheim, Germany.

2. KU Leuven-VIB Center for Brain \& Disease Research, VIB, 3000 Leuven, Belgium.

3. Department of Neurosciences, Leuven Institute for Neuroscience and Disease, KU Leuven, 3000 Leuven, Belgium.

4. Faculty of Computer Science, University of Applied Sciences Mannheim, Paul-WittsackStraße 10, 68163 Mannheim, Germany

5. Department of Psychiatry and Neurochemistry, Sahlgrenska Academy at the University of Gothenburg, Mölndal Hospital, House V3, 43180 Mölndal, Sweden

6. Department of Neuroscience, Physiology and Pharmacology, University College London, Gower Street, London WC1E 6BT, United Kingdom

7. UK Dementia Research Institute at UCL, University College London, London, UK

8. University College London Queen Square Institute of Neurology, Queen Square, London WC1N 3BG, United Kingdom

*Correspondence should be addressed to C.H. (carsten.hopf@medtech.uni-heidelberg.de)

Prof. Dr. Carsten Hopf

Mannheim University of Applied Sciences

Paul-Wittsack-Str. 10

68163 Mannheim, Germany

Phone +49 (0)621/292-6802 
Enzlein et al. Computational Analysis of Amyloid Plaque Composition Submission to Anal. Chem.

\section{ABSTRACT}
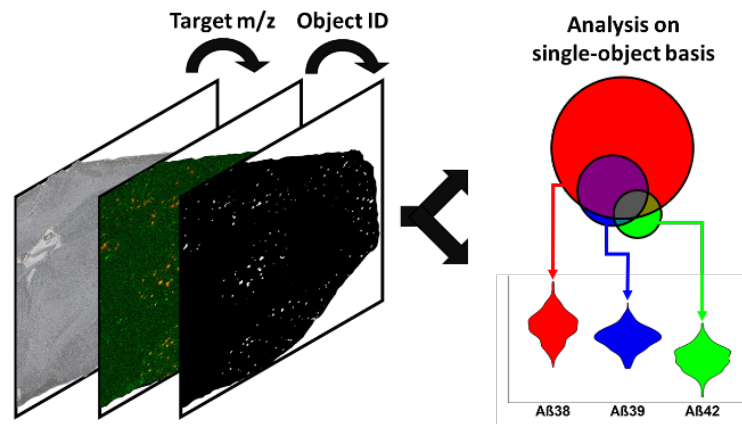

MALDI mass spectrometry imaging (MSI) enables label-free, spatially resolved analysis of a wide range of analytes in tissue sections. Quantitative analysis of MSI datasets is typically performed on single pixels or manually assigned regions of interest (ROI). However, many sparse, small objects such as Alzheimer's disease (AD) brain deposits of amyloid peptides called plaques are neither single pixels nor ROI. Here, we propose a new approach to facilitate comparative computational evaluation of amyloid plaque-like objects by MSI: a fast PLAQUE PICKER tool that enables statistical evaluation of heterogeneous amyloid peptide composition. Comparing two AD mouse models, APP NL-G-F and APP PS1, we identified distinct heterogeneous plaque populations in the NL-G-F model, but only one class of plaques in the PS1 model. We propose quantitative metrics for the comparison of technical and biological MSI replicates. Furthermore, we reconstructed a high accuracy 3D-model of amyloid plaques in fully automated fashion, employing rigid and elastic MSI image registration using structured and plaque-unrelated reference ion images. Statistical single plaque analysis in reconstructed 3D-MSI objects revealed the $A \beta_{1-42 A r c}$ peptide to be located either in the core of larger plaques or in small plaques without co-localization of other $A \beta$ isoforms. In 3D, a substantially larger number of small plaques were observed than the 2D-MSI data indicated, suggesting that quantitative analysis of molecularly diverse sparsely-distributed features may benefit from 3D-reconstruction.

\section{3 words}


Enzlein et al. Computational Analysis of Amyloid Plaque Composition Submission to Anal. Chem.

\section{INTRODUCTION}

Matrix-assisted laser desorption/ionization mass spectrometry imaging (MALDI MSI) has emerged as a key tool for label-free investigation of the spatial distribution of various molecules such as peptides, lipids, drugs, glycans, and other metabolites in 2D tissue sections (2D-MSI) [1, 2]. Recent advances in the speed of data acquisition and -processing have improved the feasibility of quantitative analyses of MSI datasets as well as image registration and reconstruction of 3D-MSI models [3-6]. However, most data analysis in current MSI is not yet based on quantitative measures but on the overall mean spectrum of either the whole dataset or of manually selected ROIs [7-9]. Any manual selection of ROIs may introduce user bias. Other studies define relevant ROIs by multivariate clustering [10] (e.g. kmeans, PCA, pLDA, or combinations thereof) or by co-registration of MSI datasets to other imaging modalities like H\&E-staining or lipid MSI data [11]. However, small sparse objects like neurodegenerative plaques in brains occur only in a small subset of spectra. This causes their under-representation or even absence in the overall mean spectrum, which may require computational signal enhancement [12]. Computational workflows designed for definition and quantitative evaluation of classes of sparse objects on the single object basis are lacking in 2D-MSI.

Moreover, most 3D-MSI studies are currently based on manual rigid 2D-alignments either of ion images or of co-registered optical tissue images $[3,5,6]$. More advanced 3D-reconstruction methods are based on automatic rigid registration of tissue stained post-MSI with H\&E, but they still use manual rigid registration of MSI data to corresponding H\&E images [4]. Recently, automatic non-linear registration of MSI data to magnetic resonance imaging data has been reported [13]. However, to extract relevant biomedical information from large MSI datasets, more efficient and custom-tailored methods for feature extraction and multivariate statistical analysis have to be developed [4, 14]. For example, computational workflows for quantitative analysis of sparse MSI-objects in 3D, which include estimates of their size, have not been reported yet.

One major hallmark of Alzheimer's disease (AD) is the formation of sparse protein deposits called amyloid plaques, consisting of various isoforms of amyloid- $\beta(A \beta)$ peptides, which originate from amyloid precursor protein (APP), and presumably other molecules such as glycosphingolipids [15-17]. Many lines of evidence support an important role of $A \beta$ peptides in the pathogenesis of $A D[18,19]$. Numerous $A \beta$ peptides with different $\mathrm{N}$ - and $\mathrm{C}$-terminal truncations and modifications like 
Enzlein et al. Computational Analysis of Amyloid Plaque Composition Submission to Anal. Chem.

pyroglutamation have been described, but most research focuses on $A \beta$ isoforms starting at position 1 , especially on $A \beta_{1-42}$ [20]. Recent studies suggest an important role of longer forms of $A \beta$ than $A \beta_{1 \text { - }}$ 42 in the etiology of AD [21-23]. Traditionally, amyloid plaques have been studied extensively using immunochemistry, which has led to a strong focus on a few well-studied $A \beta$ species such as $A \beta_{x-38}$, $A \beta_{x-40}$ and $A \beta_{x-42}$ or collective staining of multiple $A \beta$ species (total $A \beta$ ). MALDI MSI has enabled untargeted studies of $A \beta$ plaques, i.e. simultaneously analysis of many distinct $A \beta$ species. Many studies have highlighted the complex molecular composition of $A \beta$ plaques in brains of human $A D$ patients and of AD mouse models [9, 24-26]. Currently most MSI studies are still based on single experiments per experimental condition (i.e. they lack MSI replicates) or simply visually compare ion images in replicates without computational analysis. The reason for this is the notorious difficulty of quantitative MSI data analysis [27, 28].

Here, we present a new PLAQUE-PICKER approach and computational tools in $\mathrm{R}$ for analysis of sparsely distributed features (here: plaques) in tissue and for (plaque) population statistics. We paid special attention to fast analysis run times to accommodate future serial studies with substantial numbers of samples. Furthermore, we present a new registration approach for reconstructing a 3D model of amyloid plaques, and we apply these computational methods for a quantitative plaque analysis in different mouse models of $A D$.

\section{MATERIALS AND METHODS}

\section{Chemicals}

All reagents were HLPC grade. Acetonitrile (ACN) and Trifluoroacetic acid (TFA) were from Merck (Darmstadt, Germany). Dimethyl sulfoxide (DMSO) was obtained from Sigma-Aldrich (Munich, Germany). Milli-Q water ( $d \mathrm{dH}_{2} \mathrm{O}$; Millipore, Burlington, USA) was prepared in-house. sDHB (9:1 mixture of 2,5-dihydroxybenzoic acid (DHB) and 2-hydroxy-5-methoxybenzoic acid), the MALDIMS peptide calibration standard mix II and protein calibration standard mix I were from Bruker Daltonics (Bremen, Germany). Synthetic $A \beta_{1-38}, A \beta_{1-39}, A \beta_{1-40}, A \beta_{1-42}, A \beta_{1-43}$ and $A \beta_{1-45}$ were from rPeptide (Watkinsville, USA) and mixed at an equimolar concentration of $2 \mu \mathrm{mol} / \mathrm{L}$ in $\mathrm{ddH}_{2} \mathrm{O}(\mathrm{A} \beta$ calibration standard). 
Enzlein et al. Computational Analysis of Amyloid Plaque Composition Submission to Anal. Chem.

\section{Mouse models and tissue preparation for MALDI MSI}

App ${ }^{\text {tm3.1Tcs }}$ mice, also called APP NL-G-F mice, and transgenic Tg(Thy1-APPSw,Thy1-PSEN1*L166P) 21Jckr, referred to as APP PS1 mice, were described elsewhere [29-31]. APP PS1 mice harbor APP Swedish and presenilin 1 (PS1) L1166P mutations, both overexpressed using a Thy1 promotor. APP NL-G-F knock-in mice expresses the mouse APP gene with a humanized A $\beta$ sequence with Swedish, Arctic and Beyreuther/Iberian mutations under the control of the mouse endogenous promotor. This leads to cell-specific expression of APP at wild-type levels. All experiments were approved (No. 142/2015) by the Ethics Committee on Animal Experimentation of the University of Leuven. For APP NL-G-F (age 18 weeks) and APP PS1 (20 weeks) we analyzed one mouse in two independent technical replicates each. For APP NL-G-F (67 weeks) we analyzed three brains (biological replicates), of which two were additionally technically replicated (Table S1).

Fresh-frozen mouse brains were cut $(10 \mu \mathrm{m}$; CM 1950 cryostat, Leica Biosystems, Nussloch, Germany) and thaw-mounted on indium-tin-oxide (ITO)-coated glass slides (Bruker Daltonics). All slides were dried in a desiccator overnight at RT. Prior to protein MSI, tissue sections were delipidated using the washing procedure by Yang et. al. [32]: $70 \%$ ethanol (30 s), $100 \%$ ethanol (30 s), Carnoy's fluid (60/30/10 ethanol/chloroform/acetic acid v/v/v) (120 s), $100 \%$ ethanol (30 s), dd $\mathrm{H}_{2} \mathrm{O}(30 \mathrm{~s})$ and $100 \%$ ethanol (30 s). After drying, the slides were coated with eight layers of $60 \mathrm{mg} / \mathrm{mL}$ sDHB matrix in $\mathrm{ACN} / \mathrm{ddH}_{2} \mathrm{O} / \mathrm{TFA}(40 / 60 / 0.5 \mathrm{v} / \mathrm{v} / \mathrm{v})$ using an M5 Sprayer (HTX Technologies, Chapel Hill, USA) in a crisscross pattern at a flowrate of $0.02 \mathrm{~mL} / \mathrm{min}$ and a velocity of $750 \mathrm{~mm} / \mathrm{min}$. $2 \mathrm{~mm}$ Track spacing, $40 \mathrm{~mm}$ nozzle height and $30^{\circ} \mathrm{C}$ gas temperature were applied.

\section{MALDI MS Imaging}

MALDI MSI measurements were done on a Rapiflex MALDI-TOF MS (Bruker Daltonics) in positive linear mode with $\mathrm{m} / \mathrm{z}$ 2,000-10,000 using FlexImaging 5.0 software (Bruker Daltonics). The acquisition method was calibrated using a 1/1/1 (v/v/v) mix of protein calibration standard I, peptide standard II and $A \beta$ calibration standard using quadratic calibration. 250 laser shots at $10 \mathrm{kHz}$ repetition rate were accumulated for each raster spot with a lateral resolution of $20 \mu \mathrm{m}$ 
Enzlein et al. Computational Analysis of Amyloid Plaque Composition Submission to Anal. Chem.

and a spot size of $20 \times 20 \mu \mathrm{m}$. The lon Source 1 was set to $20 \mathrm{kV}$, PIE to $1.37 \mathrm{kV}$ and the ion lens to $1.75 \mathrm{kV}$ the delayed extraction time was set to $150 \mathrm{~ns}$. The digitizer was set to $0.63 \mathrm{GS} / \mathrm{s}$ and the deflector cut off mass for matrix suppression was set to $m / z 2000$.

\section{LC-MS/MS verification of $A \beta$ peptides in tissue extracts}

See Supplemental Methods

\section{MSI Data processing and 3D-reconstruction}

MSI raw data was converted to the imzML file format [33] using a converter programmed inhouse. Subsequently, the datasets were imported into R 3.4.1 (R Foundation for Statistical Computing, Vienna, Austria) using MALDIquant and MALDlquantForeign packages [34], TICnormalized, Savitzky-Golay-smoothed, and the baseline was removed using the "TopHat"method. As this method selects features on the image level, it does not need peak picking, which is usually one of the most time-consuming steps, since it is based on single spectra and does not respect spatial relationships. Nine consecutive $10 \mu \mathrm{m}$ tissue slices were prepared on a single ITO slide and measured in a single MSI run. Registration of slices and following 3D-reconstruction was done using the $\mathrm{M}^{2}$ aia tool (Cordes et. al., unpublished). Briefly, to create a 3D-MSI volume, consecutive slices were aligned to each other, applying subsequent rigid and elastic image-based registration steps. These used structural-rich ion images ( $5447 \mathrm{~m} / \mathrm{z}+-5 \mathrm{Da}$ ) for each slice. Rigid and elastic transformation parameters are stored for each alignment step and can be used to 3D reconstruct ion image volumes of target mass values. To assess individual plaque features specific for a given mouse model, area under the curves for defined $m / z$ intervals in linear MS mode corresponding to $A \beta$ peptide species were extracted (Table S2). None of these signals were observed in corresponding wild-type mice (Figure S5, Figure S6). For each ion image, an individual threshold for binarization was determined by T-point thresholding (Figure S1) [35]. In the 3D case, this was separately done for each slice and each $A \beta$ peptide species. To each of the individual binarized images, a connected component labelling process as implemented in the raster package (vers. 3.0-7; https://CRAN.R-project.org/package=raster) or in the package neuroim (https://CRAN.R-project.org/package=neuroim) for 3D was applied [35, 36]. By combining pixel 
Enzlein et al. Computational Analysis of Amyloid Plaque Composition Submission to Anal. Chem.

sets thus extracted for each individual ion image (= A $\beta$ species), a unified collection of pixel sets was generated by joining pixel sets with common pixel coordinates. This process results in a unique ID assigned to each plaque giving access to summary statistics and the individual spectra themselves.

\section{RESULTS}

\section{Single object-based feature extraction from 2D-MALDI-MSI datasets for computational analysis of amyloid plaque composition}

Amyloid plaques and other objects of interest in MSI (like infiltrating immune or tumor cells) are sparsely distributed and heterogeneously composed, which can make data analysis challenging. Common immunohistochemical approaches can only evaluate a small number of $A \beta$ peptide species, and concerns about antibody specificity are valid. Therefore, much can be learned about regional differences in $A \beta$ peptide composition of amyloid plaques from the statistical evaluation of MALDI images of brains from AD mouse models and eventually from translation to human brain samples. Commonly used MSI data analysis workflows either rely on segmentation algorithms like k-means or on manual user input for selection of ROls. Both options are suboptimal, as k-means is not well suited for imbalanced group sizes and densities, which is a common property of amyloid plaque MSI data, whereas manual data analysis is time-consuming and can introduce user bias, which may require mitigation by multiple expert users or multi-site studies [37-39].

To this end, we developed a fast and simple workflow in $\mathrm{R}$ for selection of molecularly diverse and sparsely-distributed features that enables molecular analysis on a single plaque level and thereby population statistics (Figure S1): First, a number of $\mathrm{m} / \mathrm{z}$ features is selected from a lookup table, and the corresponding ion images are extracted from the dataset. Pixels containing $A \beta$ peptide signals are then selected by binarizing their ion-images based on individual thresholds [35]. Adjacent Spectra/pixels are assigned a unique ID, which links all spectra that the plaque is composed of. Importantly, the number of spectra per ID provides an estimate for plaque size. This PLAQUE PICKER workflow enabled computational evaluation of the molecular composition of 
Enzlein et al. Computational Analysis of Amyloid Plaque Composition Submission to Anal. Chem.

amyloid plaques and correlation of molecular composition with other plaque features such as size. Because of fast run times, it is possible to process many MSI datasets within a short amount of time.

\section{Plaques in APP NL-G-F mice are heterogeneous in composition whereas APP PS1 bears a homogenous plaque population}

Using the PLAQUE PICKER workflow, we compared two mouse models of AD, APP PS1 at age 20 weeks (20w) and APP NL-G-F ( 18w and 67w; Table S1). In APP PS1 mice, amyloid plaque pathology is driven by APP overexpression and mutations (see methods for details) known to increase total $A \beta$-production $[40,41]$ and to preferably produce $A \beta_{1-42}$ over $A \beta_{1-40}[30]$. In contrast, the APP NL-G-F mouse model is a knock-in of a triple-mutant APP (see methods) with cell-type specific APP expression at wild-type levels and presumably devoid of artifacts originating from overexpression [29]. Mutations promote $A \beta$ aggregation through oligomerization and reduced proteolytic degradation [42, 43]. A $\beta$ peptides in APP NL-G-F mice contain the Arctic (E693G) mutation and are denoted as $A \beta_{x-y A r c}$. We investigated the differences in plaque composition between brains of these mouse models to exemplify the utility of the data processing workflow.

We subjected cryosections of the respective mouse brains to MALDI-TOF-MSI in linear positive mode using sDHB as matrix. Presence of the $A \beta$ peptides assigned after MSI was verified by LCMS/MS analysis of homogenates from brain slices adjacent to those used for MSI (Table S5 - S7). Amyloid peptides $A \beta_{1-38 A r c,} A \beta_{1-39 A r c,} A \beta_{1-40 A r c} A \beta_{1-42 A r c}$ and $A \beta_{1-40}, A \beta_{1-42}, A \beta_{1-43}$ were detected by MSI and verified by LC-MS/MS in the mouse models APP NL-G-F and APP PS1, respectively. Presumably owing to its low abundance, $A \beta_{1-38}$ in APP PS1 mice was detected by MSI (assigned based on sparse distribution, plaque-like morphology and co-localization with verified A $\beta$ species) but not by LC-MS/MS.

In two replicate MALDI ion images of $A \beta$ peptides in adjacent tissue sections of an 18w APP NL-G-F mouse brain, $A \beta_{1-38 \text { Arc }}$ was present in small plaques in cortex and a few larger plaques in thalamus, whereas $A \beta_{1-42 A r c}$ formed mostly smaller plaques in hippocampus but also in cortex and thalamus 
Enzlein et al. Computational Analysis of Amyloid Plaque Composition Submission to Anal. Chem.

where it co-localized with $A \beta_{1-38 A r c}$ (Figure S2). No assigned $A \beta$ peptide species were detected in the age-matched control mice (Figure S5). Plaque pathology was much more pronounced in 67w than in 18w APP NL-G-F brain: Intensity, size and number of plaques were all drastically higher (Figure S3). Based on the number of associated pixels and overall intensity, the most abundant

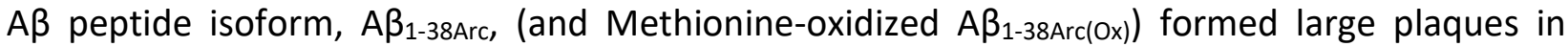
cortex, hippocampus and thalamus and co-localized with $A \beta_{1-39 A r c}$ (and low-abundance $A \beta_{1-40 A r c}$ ). In contrast, $A \beta_{1-42 A r c}$ formed smaller plaques that mostly but not exclusively co-localized with $A \beta_{1-}$ 38Arc. Interestingly, $A \beta_{1-42 A r c}$ co-localizing with the other $A \beta$ peptide isoforms typically appeared in the plaque core. All these findings were consistent across three separate brains with a total of five MSI technical replicates (Table S1). In striking contrast with APP NL-G-F brain, plaque composition in two technical replicates of the 20w APP PS1 mouse was very uniform (Figure S4), displaying co-localizing abundant $A \beta_{1-43}$ and $A \beta_{1-42}$ and low-abundance $A \beta_{1-40}$ and $A \beta_{1-38 \text {, }}$ confirming the known shift of the $A \beta$ peptide products towards longer species in PS1 mice [44].

The computational workflow added detail and quantitative metrics for quality assurance to this initial evaluation: Plaque population-based statistics of the $A \beta_{1-42} / A \beta_{1-38}$ and $A \beta_{1-42 A r c} / A \beta_{1-38 A r c}$ ratios revealed more $A \beta_{1-42}$-rich plaques in APP PS1 brain than $A \beta_{1-42 A r c}$-rich plaques in $18 w$ and

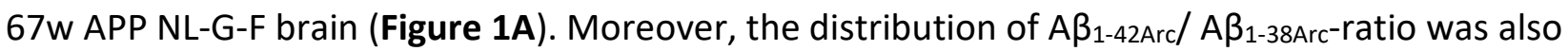
more unequal in APP NL-G-F mice than in the APP PS1 mouse, as demonstrated by a higher Gini coefficient (Figure 1B). Plaque population-based statistics of amyloid composition highlighted surprisingly little variability between MALDI imaging experiments conducted on different slices (Figure 1C-I). For example, in the $18 \mathrm{w}$ NL-G-F brain the majority of plaques contained only $A \beta_{1-42 A r c}$ (40\% and $68 \%$ in two independent MSI experiments), whereas $13.5 \%$ and $5 \%$, respectively,

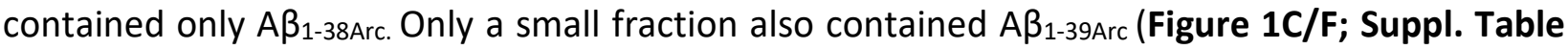

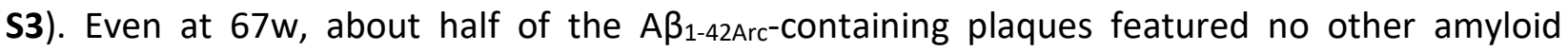

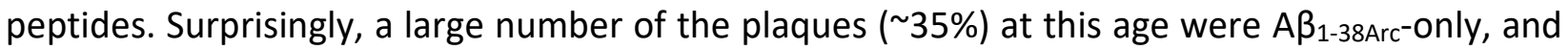
$A \beta_{1-39 A r c}$ was consistently associated with $A \beta_{1-38 \text { Arc }}$ (Figure 1 D/G). 
Enzlein et al. Computational Analysis of Amyloid Plaque Composition Submission to Anal. Chem.
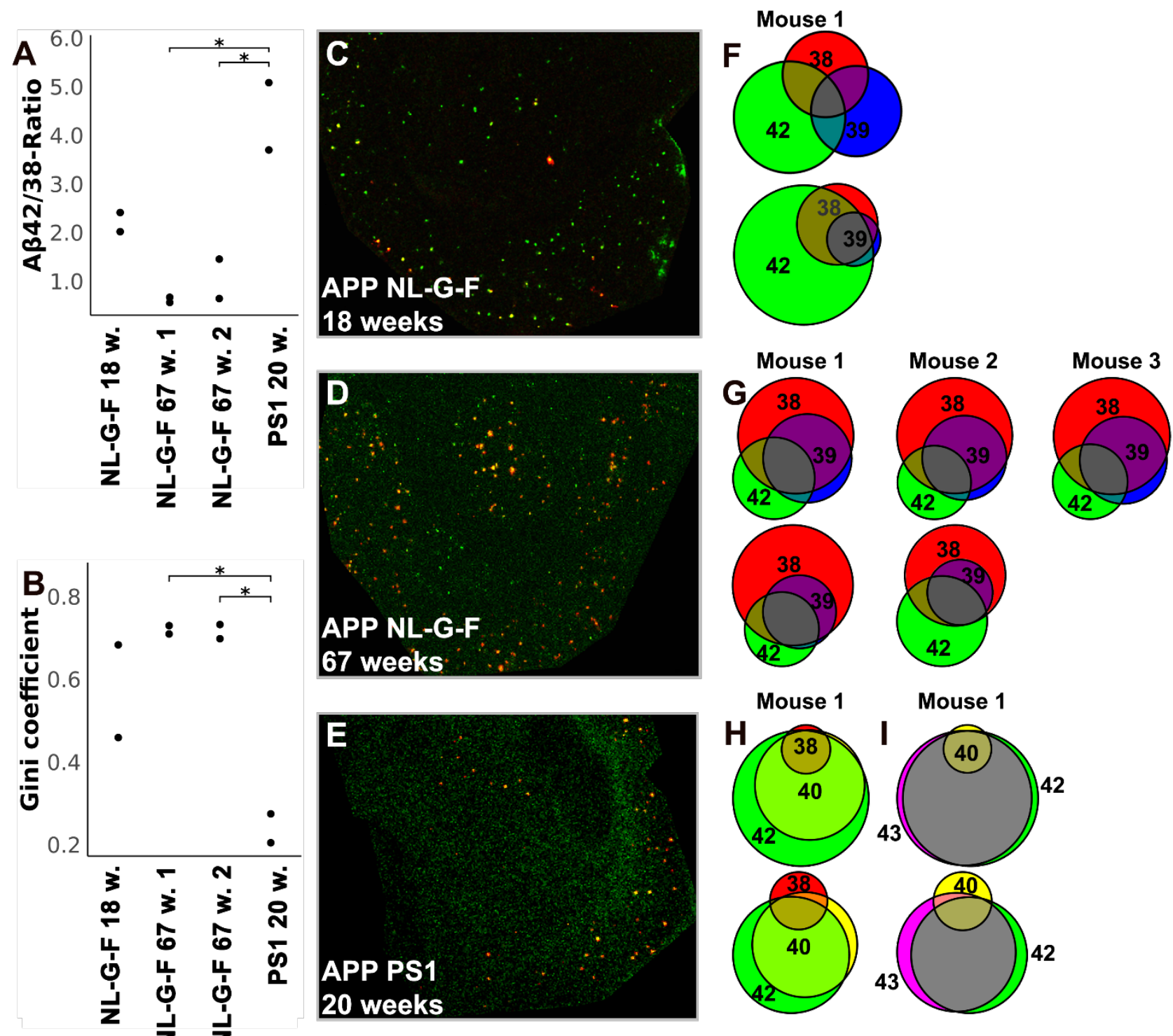

Figure 1. Quantitative analysis of amyloid plaque composition reveals differences between APP NL-G-F- and APP

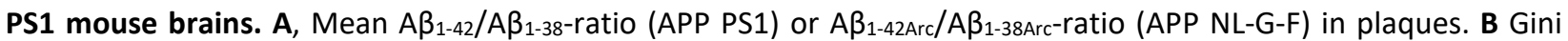
coefficient as a measure of inequality of $A \beta_{1-42} / A \beta_{1-38}$-ratios across all plaque individuals of a given brain slice (see Figure S8). Independent MSI experiments: APP NL-G-F 18 weeks $n=2$, APP NL-G-F 67 weeks $n=5$, APP PS1 $n=2$. C-I, Composition of amyloid plaques is much more uniform in APP PS1 brain than in APP NL-G-F brain. C-E, lon images of $A \beta_{1-42 A r c}(C / D)$ or $A \beta_{1-42}(E)$ in green, $A \beta_{1-38 A r c}$ or $A \beta_{1-38}$ in red. I-F, Venn-diagrams visualizing the degree of colocalization of plaque populations defined by different $A \beta$ peptide species relative to the total number of plaques: $A b_{1-38 A r c}$ (red), $A b_{1-39 A r c}$ (blue), $A b_{1-40}$ (yellow), $A b_{1-42}$ and $A b_{1-42 A r c}$ (green), Ab 1-43Arc (purple). $C \& F$ APP NL-G-F mouse (18 weeks) D \& G APP NL-G-F mouse (67 weeks) E, H \& I APP PS1 mouse (20 weeks). See Table S 3 for a full overview of the co-localization of $A \beta$ peptide species. 
Enzlein et al. Computational Analysis of Amyloid Plaque Composition Submission to Anal. Chem.

Again, plaque composition was remarkably consistent between five MSI experiments using three separate mice/brains. In contrast to APP NL-G-F mice, plaque composition was much more homogeneous is $20 \mathrm{w}$ APP PS1 brain, as the vast majority of plaques contained $A \beta_{1-38,} A \beta_{1-40,} A \beta_{1-42}$ and $A \beta_{1-43}$ (Figure $\left.1 \mathrm{E} / \mathrm{H} / \mathrm{I}\right)$.

\section{Large plaques in APP NL-G-F mice are associated with a greater A $\beta$-diversity and enriched in $A \beta_{1-38 A r c}$}

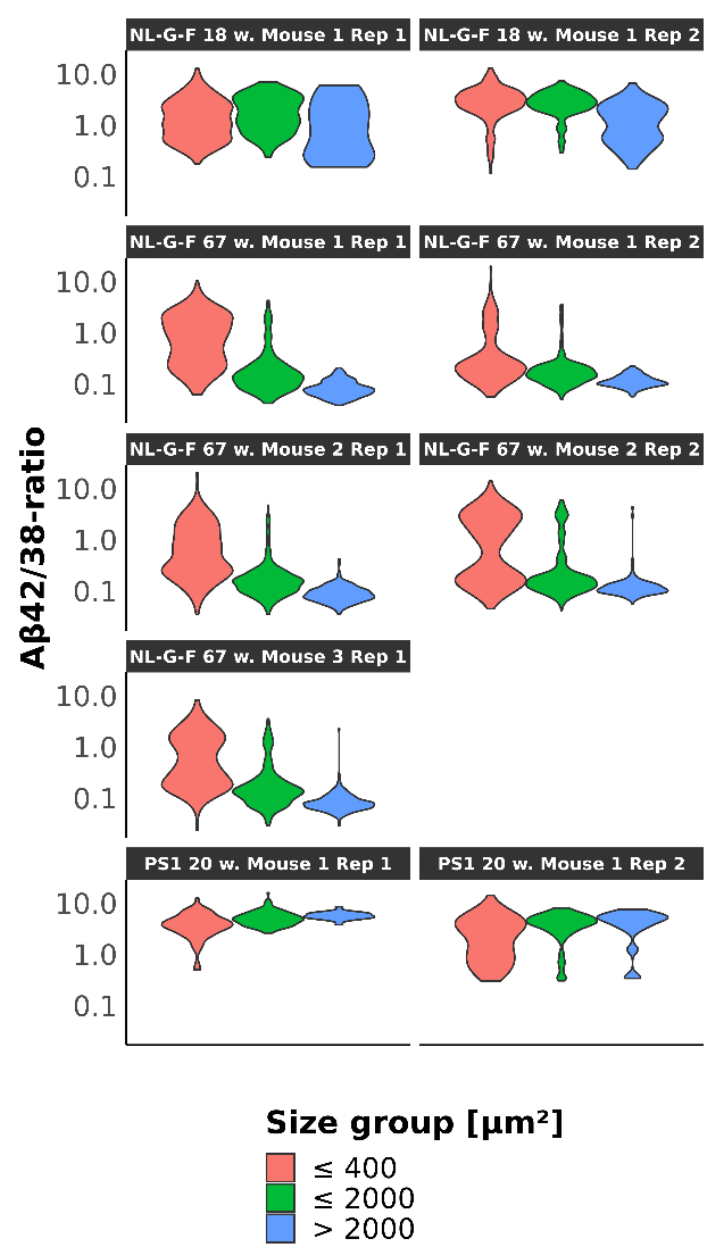

Figure 2. Differences in molecular plaque composition in two $A D$ animal models. $A \beta_{1-42} / A \beta_{1-38}$-ratio (APP PS1 brain) or $A \beta_{1-42 A r c / A} A \beta_{1-38 A r c}$-ratio (APP NL-G-F brain) in relation to estimated plaque sizes ( $\leq 400 \mu \mathrm{m}^{2}$ (red) corresponds to 1 pixel, $\leq 2000 \mu \mathrm{m}^{2}$ (green) corresponds to $\leq 5$ pixels, $>2000 \mu \mathrm{m}^{2}$ (blue) corresponds to $>5$ pixels). In APP NL-G-F mice, size-dependent differences in plaque composition increase with age, and large plaques are $A \beta_{1-38 A r c}$-rich. Plaque composition is more uniform in APP PS1 than in APP NL-G-F mouse brain. 


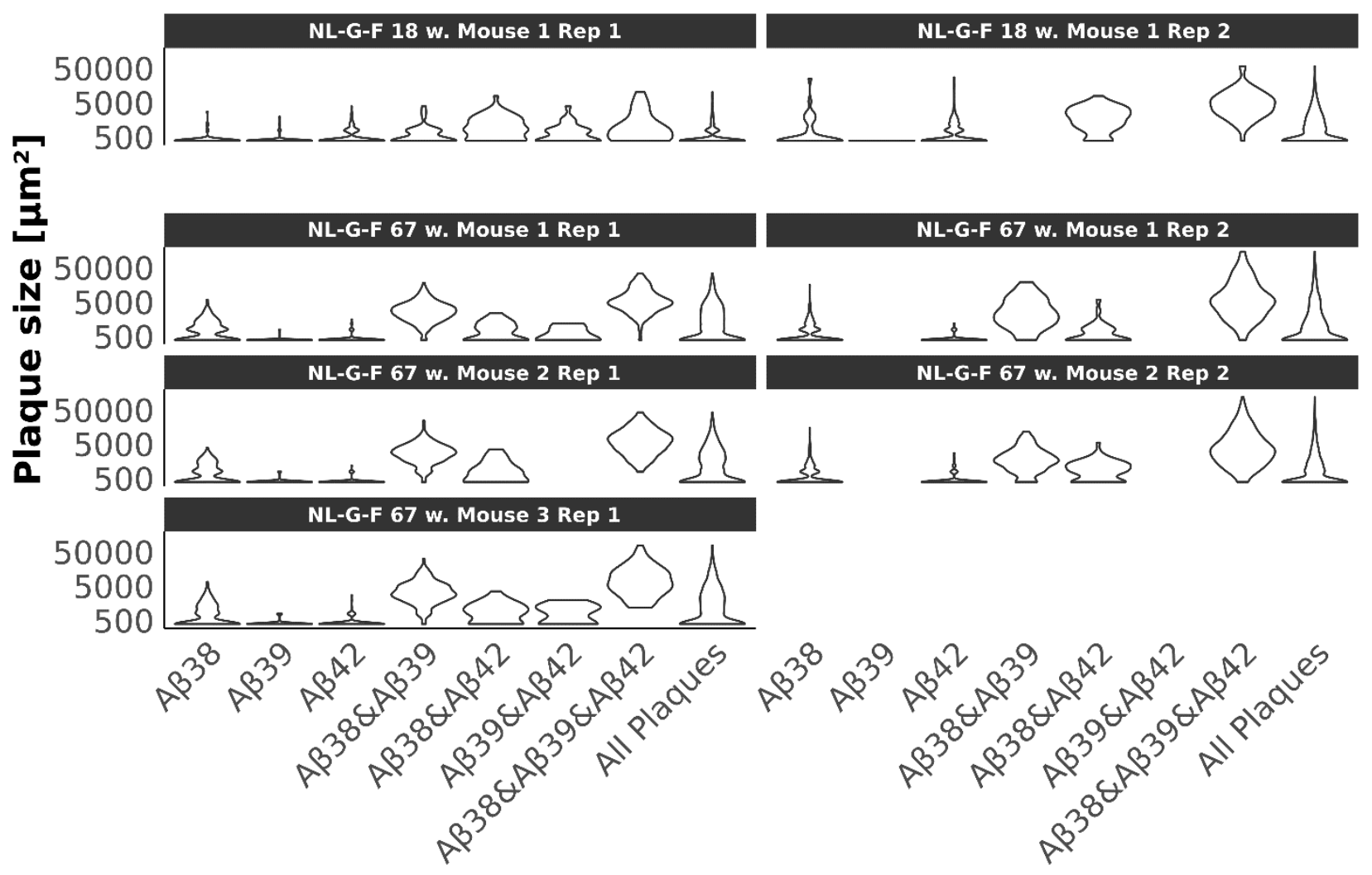

Figure 3. Plaques with higher $\mathbf{A} \boldsymbol{\beta}$-diversity tend to be larger. Molecular plaques composition versus plaque area of APP NL-G-F mice at ages 18 and 67 weeks. Plaques were split into multiple classes with distinct amyloid compositions (see Venn-diagrams in Figure 1). Size distributions of all plaques of a given molecular composition were plotted as violin-plots normalized to equal maximal width of distribution.

To add further detail to the analysis of amyloid plaque composition, we divided plaques into three different size groups ("small plaques" $\leq 400 \mu \mathrm{m}^{2}$ corresponding to 1 pixel, "medium plaques" of $400-2000 \mu \mathrm{m}^{2}$ corresponding to $\leq 5$ connected pixels, and $>2000 \mu \mathrm{m}^{2}$ corresponding to $>5$ pixels: "large plaques") (Figure 2). In young APP NL-G-F mice, large plaques displayed a wide range of $A \beta_{1-42 A r c} / A \beta_{1-38 \text { Arc }}$ ratios, whereas small and medium plaques had mostly high $A \beta_{1-42 A r c} / A \beta_{1-38 \operatorname{Arc}}$ ratios. In old APP NL-G-F mice, however, large plaques featured low $A \beta_{1-42 A r c} / A \beta_{1-38 A r c}$ ratios and small plaques a wider range of $A \beta_{1-42 A r c} / A \beta_{1-38 A r c}$ ratios. In contrast, in APP PS1 mice differences between the different size groups were much smaller for all A $\beta$-ratios. For the APP PS1 mouse there is a tendency that larger plaques have a higher $A \beta_{1-42} / A \beta_{1-38}$ (Figure 2) whereas the ratio for $A \beta_{1-42} / A \beta_{1-40}$ and $A \beta_{1-43} / A \beta_{1-40}$ seem to be unaffected by size of the considered plaque (Figure S9). 
Enzlein et al. Computational Analysis of Amyloid Plaque Composition Submission to Anal. Chem.

Apparently, amyloid composition was very heterogeneous in APP NL-G-F mouse brain, but less so in APP PS1 brain. Also, we observed a strong trend suggesting that larger plaques featured lower

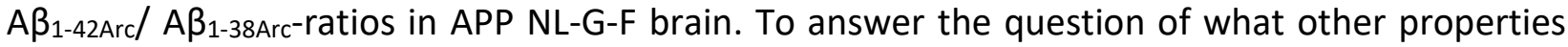
distinguished larger from smaller plaques in the APP NL-G-F mouse model, we separated all plaques into different classes depending on the $A \beta$-species associated with them (see Venndiagrams in Figure 1) and analyzed their estimated size (Figure 3). We found that plaques with a higher $A \beta$-diversity were associated with larger plaques. Especially plaques containing $A \beta_{1-38 A r c}$ (alone or in combination with other A $\beta$-species) were consistently larger, especially in old APP NLG-F mice.

\section{High-resolution 3D-model of amyloid plaques in APP NL-G-F mouse model}

We wanted to further investigate the non-uniform plaque pathology of the APP NL-G-F mouse. Here the main motivation was to examine if the observation that $A \beta_{1-42 A r c}$ typically composes the core of a plaque holds true or if it was just caused by the misleading nature of 2D-images, i.e. sections from 3D-volumes. After all, the cutting plane may have been placed in the center of the volume (in which case a statement about the core of a plaque would be possible) or at the periphery of a larger volume ("tip of the iceberg effect"). We therefore chose to reconstruct a 3Dmodel of the 67w APP NL-G-F brain. We measured nine consecutive $10 \mu \mathrm{m}$-slices, all mounted on the same slide, in a single MSI run at $20 \times 20 \mu \mathrm{m}$ resulting in a voxel volume of $20 \times 20 \times 10 \mu \mathrm{m}$. We then reconstructed the 3D model in an automated approach using the M2aia IT tool that is currently being developed. We used the ion image of $\mathrm{m} / \mathrm{z} 5447 \pm 5 \mathrm{Da}$ as reference landmark for all slices, as it represented the general brain structures like cortex and hippocampus well.

At first glance, the overall plaque distribution was similar in 2D- and 3D images of the 67w APP NL-G-F brain: As in 2D, the highest plaque density was in cortex and hippocampus also in 3D, and


medium-sized plaques extended over several z-layers (Figure 4B/C). These plaques were typically

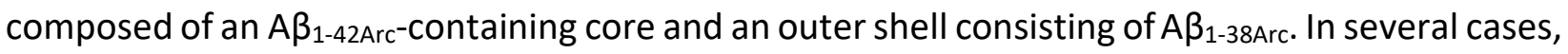

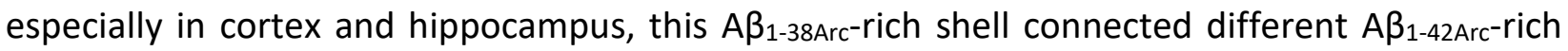


Enzlein et al. Computational Analysis of Amyloid Plaque Composition Submission to Anal. Chem.

cores (Figure $4 \mathrm{C}$ ). As in the 2D-comparison, the APP NL-G-F (but not the APP PS1) mouse

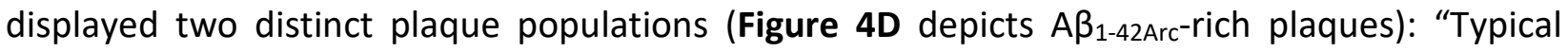
plaques" consisting of $A \beta_{1-38 A r c}$ and $A \beta_{1-39 A r c}$ (with some $A \beta_{1-40 A r c}$ and $A \beta_{1-42 A r c}$ ) were located in cortex and hippocampus and much larger than the second, small and $A \beta_{1-42 A r c-r i c h}$ plaque population. An animated version of the 3D-model is available as supplementary content.
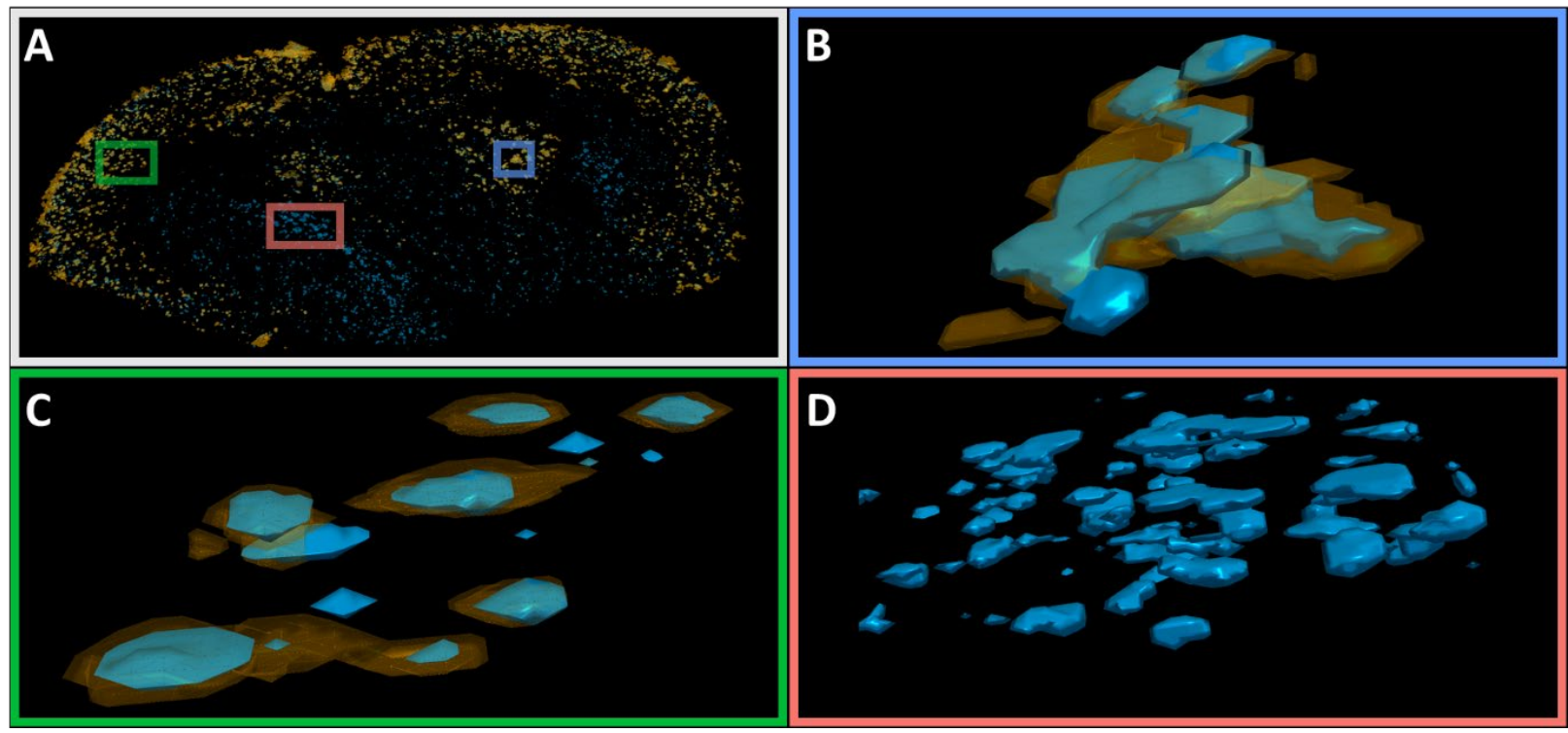

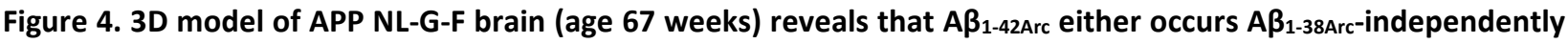
or at the core of larger plaques, surrounded by $\mathbf{A} \boldsymbol{\beta}_{1-38 \text { Arc }}$. Orange: $A \beta_{1-38 A r c}$, Blue: $A \beta_{1-42 A r c}$. , Overview of the whole 3D-model representing $\sim 200 \mu \mathrm{m}$ section of the brain (see supplementary material for an animated version). Colored rectangles mark positions of zoomed in plaques in C-D. B, Example of a larger plaque extending over several z-layers and with $A \beta_{1-42 A r c}$ in several inner regions connected by $A \beta_{1-38 A r c .} \mathbf{C}$, Example of typically sized plaques. $A \beta_{1-42 A r c}$ is

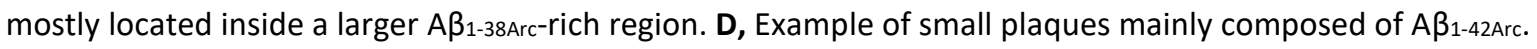

This second plaque population was located in brain regions populated also by "typical plaques", but it was also found in regions devoid of "typical plaques" like the hypothalamus. Overall, the 3D model presented a much more detailed overview of plaque morphology and the distribution of $A \beta$ peptides inside them. We therefore applied the method for single-plaque statistics to the 3D model and compared results for a single slice to a full 3D-stack. When the single slices used for the reconstruction of the 3D model were evaluated individually in 2D (i.e. as additional technical replicates), results agreed with Figure 2 (Figure S10). 

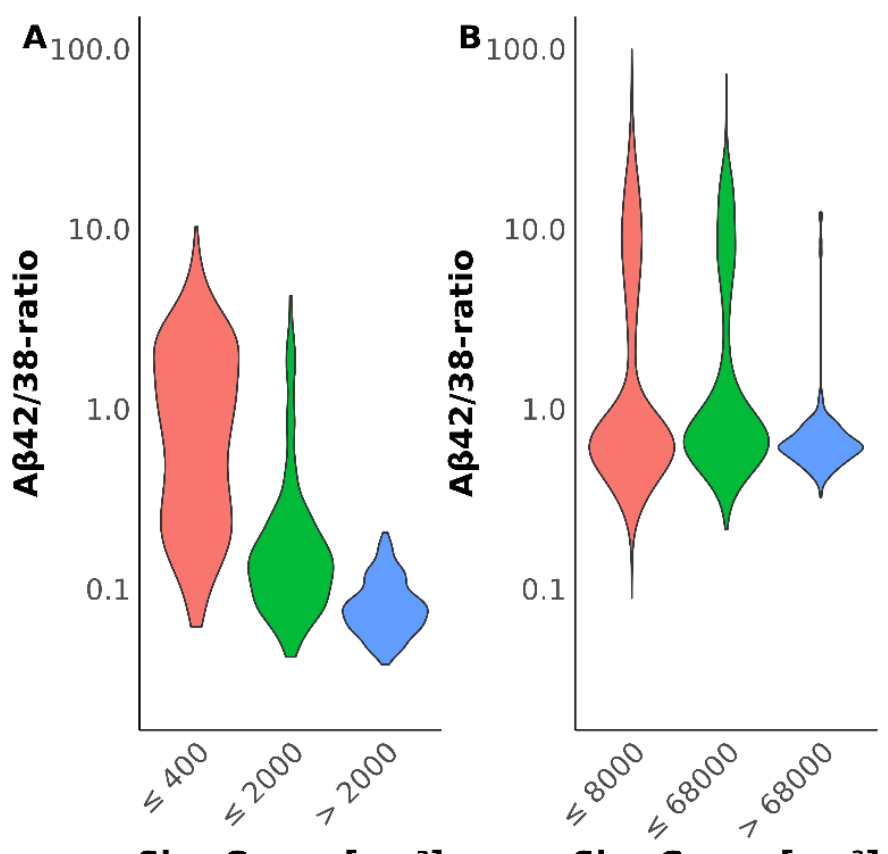

Figure 5. Statistical evaluation of the relationship between single-plaque $A \beta_{1-42 A r c} A \beta_{1-38 A r c}$-ratios vs. plaque area/volume of APP NL-G-F mouse (67 weeks) in 2D and 3D. A, 2D-evaluation of a single $10 \mu \mathrm{m}$ slice. B, 3Devaluation of 9 consecutive slices at $10 \mu \mathrm{m}$ thickness.

For comparison of $A \beta_{1-42 A r c /} A \beta_{1-38 A r c}$-ratios in a single slice of $67 w$ APP NL-G-F brain (Figure 5A) to the 3D-model (Figure 5B), the size bins for plaque areas used in the 2D case were converted to volumes under the assumption of spherical plaques. In contrast to 2D-statistics, the majority of plaques of all sizes displayed an $A \beta_{1-42 A r c /} A \beta_{1-38 A r c}$-ratio just below 1 , whereas a smaller fraction of plaques were $A \beta_{1-42 A r c}-r i c h$ (Figure 5B). More small plaques were $A \beta_{1-42 A r c}-r i c h$ than for any other size class, but the trend was notably weaker than in 2D-statistics. This could be replicated in a second independent 3D-model based on MSI for different tissue sections (Figure S11). This observation may support a "tip of the iceberg" notion for 2D-based statistics of 3D-objects and may suggest that biological phenomena should be evaluated in 3D more often also in the field of MSI. 
Enzlein et al. Computational Analysis of Amyloid Plaque Composition Submission to Anal. Chem.

\section{DISCUSSION}

In this study, we presented a fast technique for image-wide feature extraction of sparse, small and separated objects and statistical tools for evaluation and comparison of relative abundance, co-localization and sizes of structures in 2D and in 3D. Our computational workflows were applied to statistical evaluation of amyloid plaques in brains of $A D$ model mice, but they should be generally applicable for the statistical analysis of sparsely distributed objects in MSI. Current software solutions widely used throughout the MSI community regularly use hierarchical- or kmeans-clustering as a means for segmentation or ROI selection. For many applications this may lead to good results, and scalability and simplicity of the algorithms enables fast runtimes. A common property of MSI images of plaques in $A D$ brains (but also of other analytical topics in MSI, e.g. rare cell types in an organ [45]) is the low number of spectra, which carry AD-associated signals (or signals of certain rare cell types in other applications). In addition, the intensity of $A \beta$ signals can vary by $1-2$ orders of magnitude, and typically also very low signals (but with SNR $\geq 3$ ) may be selected. This leads to unequal group sizes (signal-baring spectra/pixels vs. non-signalbaring spectra) and unequal group densities (all spectra with $A \beta$ signals SNR $<3$ meaning "no plaque" vs. all spectra with SNR $\geq 3$ meaning "plaque"). Both of these properties (unequal group sizes and densities) are known weaknesses of k-means algorithms [46]. We have therefore chosen a new approach that focuses on the sparsity of signals.

As peptide MSI of AD brains means targeted analysis of known $m / z$ values of $A \beta$ isoforms, the actual segmentation task can be better described as separation of fore- and background on a relatively low number of ion-images. This is reminiscent of immunohistochemistry image analysis. Many typical images consist of several sets of coherent pixels with comparable grey levels, which often results in histograms with at least two modes, where a threshold can be set at the local minima between modes [47]. However, ion images of sparse signals like plaques tend to be unimodally distributed, and therefore we used T-point thresholding [48], which results in binary ion images of the $\mathrm{m} / \mathrm{z}$ of interest (i.e. A $\beta$ species). By applying connected component labeling to the binary image, each plaque gets its own unique ID that raw spectra are linked with. As the number of spectra for each ID and the MSI spot size are known, the plaque size can be 
Enzlein et al. Computational Analysis of Amyloid Plaque Composition Submission to Anal. Chem.

approximated and used as an additional feature that has not been accessible for MSI in an automated way until now.

Since amyloid plaques are 3D-objects, we investigated possible observational bias caused by not considering the $3^{\text {rd }}$ dimension. To address this, we reconstructed a 3D-model of AD plaques. Although the effort is still high, very fast MALDI-imaging instruments and automated MSI image registration tools (Cordes et al., $\mathrm{M}^{2}$ aia registration tool, manuscript in preparation) should enable regular 3D analysis. 3D-amyloid plaques have been shown in several studies with different modalities like synchrotron X-ray, stimulated emission depletion microscopy or light sheet microscopy [49-51] but to our knowledge this is the first time a 3D model of AD plaque pathology has been reconstructed that enables statistical analysis of molecular details such as plaque composition.

Our 3D-model (APP NL-G-F mouse; 67w) revealed large plaques harboring serval $A \beta_{1-42 A r c-r i c h}$

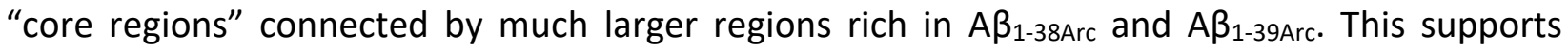
current views in the $A D$ field where longer peptides, even at low concentrations, seed the aggregation of more abundant peptides. Such a seed mechanism has been proposed for human $A D[52,53]$. However, even though the 3D-model may provide circumstantial evidence, it cannot resolve the order of events. Earlier (2D) MSI studies reported similar observations in the TgAPPswe mouse model, where they found high levels of $A \beta_{1-42}$ in younger plaques whereas plaque maturation was characterized by a relative increase in $A \beta_{1-40}$ [26]. On the other hand in another study on the TgAPPswe mouse $A \beta_{1-40}$ was found in the core of mature plaques, and $A \beta_{1-}$ 42 appeared more in diffuse plaques or diffuse radial structures of cored deposits [54]. However, the TgAPPswe model is difficult to compare, since it is based on APP overexpression and only harbors one of its three mutations of the NL-G-F mouse model. Furthermore, 3D plaque statistics done on two independent 3D-reconstructions indicates that (statistical) evaluation of 3D objects based on 2D sections might create a bias that obscures the real nature of the 3D object. This may result from the fact that large objects likely extend throughout several z-planes, whereas small objects only cover one or two z-planes. Consequently, the number of small plaques will rise with each additional z-plane considered, whereas the number of large plaques rises more slowly in 3D 
Enzlein et al. Computational Analysis of Amyloid Plaque Composition Submission to Anal. Chem.

than in 2D. In addition, small parts of large plaques will still be considered a small plaque in 2D, whereas the $3^{\text {rd }}$ dimension will make such errors less likely.

So far the vast majority of published MSI studies of AD brains have presented qualitative results, i.e. investigations of presence and (co-)localization of $A \beta$ peptides on an image level (i.e. based on the overall mean spectrum) or for a manually selected subset of plaques [7-9]. Some studies have also evaluated the relative abundance of various peptide species, but also there the analysis has always been based on a subset of plaques or has evaluated them as all pixels containing signals originating from AD-associated molecular species [55]. In our single plaque analysis of APP NL-G-F mouse brain, $A \beta_{1-40 A r c}$ was a minor signal, both in overall intensity and number of signal-

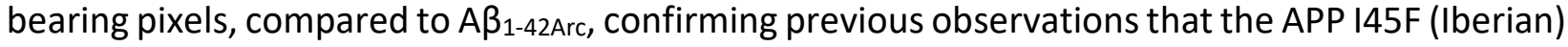
mutation causes a high $A \beta_{1-42 A r c /} A \beta_{1-40 A r c}$-ratio $[42,56]$. We found $A \beta_{1-38 A r c}$ as the main species in $N L-G-F$, but cannot be sure if this may be a result of better ionization of $A \beta_{1-38 \operatorname{Arc}}$ over $A \beta_{1-42 \operatorname{Arc}}$ (for peptide standards about 5-fold). This also means that an $A \beta$ ratio of 1 does not imply equimolarity. Because of the high complexity of the tissue, the lack of isotope-labeled standards and the intricacies of the MALDI process itself, we presently do not know the real molecular ratios in plaques.

Furthermore we introduced size estimates extracted from $\mathrm{m} / \mathrm{z}$ images as an additional feature for interpretation of MSI data of sparse objects (e.g. plaques). We used a combination of plaque composition and corresponding sizes to show that larger plaques were associated with a high diversity in plaque composition and that presence of $A \beta_{1-38 \text { Arc }}$ was associated with larger plaques.

In APP PS1 brain we revealed high abundance of a longer $A \beta$ peptide, $A \beta_{1-43}$, by MALDI MSI for the first time. Long $A \beta$ isoforms, in particular longer ones than $A \beta_{1-43}$, have recently been implicated in the pathogenesis of AD [22]. Plaque composition was more homogenous in APP PS1than in APP NL-G-F brain of similar age. It is presently unknown if this homogeneity in APP PS1 brain may be a result of APP overexpression, which also leads to other effects like perturbed axonal transport or overexpression of other APP derived fragments such as SAPP, CTF- $\beta$ and AICD that themselves also possess biological functions [29]. In contrast, the APP NL-G-F mouse of similar age displayed two types of plaques that were even more pronounced at higher age. The 
Enzlein et al. Computational Analysis of Amyloid Plaque Composition Submission to Anal. Chem.

1716F (Iberian) mutation of APP was implicated in producing abundant diffuse amyloid plaques mainly composed of $A \beta_{1-42}$ [56]. Other observations on differentially composed plaques as distinct populations were also observed in human sporadic cases of AD by MSI, but there the shorter species $(<42)$ accumulated mostly in blood vessels [9]. Taken together, the PLAQUE PICKER analytical workflow may help to resolve functional differences between animal models of $A D$ and elucidate differences between animal models and human AD.

\section{CONCLUSION}

Evaluation on single-sparse-object basis enables in-depth analysis of MSI data for e.g. AD pathology and enables better comparability for technical and biological replicates related to such analytical questions. We developed a fast analysis pipeline that enables such automated analysis for the first time in MSI. We could show different aspects like size-related differences in composition of plaques that were not assessable with classical analysis methods in MSI. Furthermore, we showed an elastically reconstructed 3D-MSI model of AD pathology and used our analysis pipeline to point out possible shortcomings of 2D based evaluation of molecularly diverse sparsely-distributed features.

\section{ASSOCIATED CONTENT}

\section{Supporting Information}

The Supporting Information is available free of charge on the ACS Publications website.

- Supplemental Methods: LC-MS/MS verification of $A \beta$ peptides in tissue extracts,

- Supplemental Figures:

- Schematic overview of the data evaluation process,

O Ion-images $A \beta$-species for different mouse models,

- Skyline spectra for different mouse models, 
Enzlein et al. Computational Analysis of Amyloid Plaque Composition Submission to Anal. Chem.

- Ion images corresponding to $\mathrm{m} / \mathrm{z}$ of $A \beta$-species in Wild-type mouse,

- Full overview of all mean $A \beta$-ratios per mouse model,

- Full overview of all Gini-values per mouse model,

- Ratios of various amyloid peptide isoforms in plaques (A $\beta$-ratio) in relation to their corresponding size bin for different mouse models of $A D, A \beta_{1-42 A r c} / A \beta_{1-38 \text { Arc-ratio }}$ of plaques in relation to their corresponding size bin of 9 individually analyzed slices of the 3D model, Comparison of size-related differences in plaquecomposition of replicated 3D models, Variations of size-bins and their impact on $A \beta_{1-42 A r c} A \beta_{1-38 A r c}-$ ratio of plaques in relation to their corresponding size bin, Molecular composition of Plaques vs plaque area of PS1 mouse at age 20 weeks, MS/MS mass spectra used for verification of $A \beta$-species, Animals included in study, Sequences and masses of $A \beta$-species, Numerical values representing the degree of co-localization of plaque populations, Assignment of observed peaks in mouse models brain using MALDI imaging MS and LC-MS/MS based verification from whole brain extracts, Animated version of 3D model (AVI).

\section{Acknowledgements and Funding}

C.H. is grateful for the support by the Hector-Foundation II (MALDI-Imaging mass spectrometer) and by the Klaus-Tschira Foundation (project MALDISTAR). C.H. and I.W. acknowledge funding by the Federal Ministry of Education and Research (BMBF; FH-Impuls Partnerschaft $\mathrm{M}^{2}$ Aind; Project: M²OGA; Förderkennzeichen 13FH8I02IA). This study was also supported by the Stichting Alzheimer Onderzoek (SAO; S16013) and the FWO research project (GOB2519N) to L.C.G. The Swedish Research Council VR (\#2019-02397, \#2018-02181); the Swedish Alzheimer Foundation (Alzheimerfonden), Alzheimer Research UK and the Åke Wiberg Foundation are acknowledged for financial support to J.H. Stiftelsen Gamla Tjänarinnor, Stohnes Stiftelse, and Swedish Dementia Foundation (Demensfonden) are acknowledged for financial support of J.H. and W.M. All authors confirm no competing financial interests. 
Enzlein et al. Computational Analysis of Amyloid Plaque Composition Submission to Anal. Chem.

\section{LITERATURE}

[1] S. Schulz, M. Becker, M.R. Groseclose, S. Schadt, C. Hopf, Advanced MALDI mass spectrometry imaging in pharmaceutical research and drug development, Curr Opin Biotechnol 55 (2019) 5159.

[2] K.O. Schubert, F. Weiland, B.T. Baune, P. Hoffmann, The use of MALDI-MSI in the investigation of psychiatric and neurodegenerative disorders: A review, Proteomics 16(11-12) (2016) 1747-58.

[3] J. Quanico, L. Hauberg-Lotte, S. Devaux, Z. Laouby, C. Meriaux, A. Raffo-Romero, M. Rose, L. Westerheide, J. Vehmeyer, F. Rodet, P. Maass, D. Cizkova, N. Zilka, V. Cubinkova, I. Fournier, M. Salzet, 3D MALDI mass spectrometry imaging reveals specific localization of long-chain acylcarnitines within a 10-day time window of spinal cord injury, Sci Rep 8(1) (2018) 16083.

[4] N.H. Patterson, M. Tuck, R. Van de Plas, R.M. Caprioli, Advanced Registration and Analysis of MALDI Imaging Mass Spectrometry Measurements through Autofluorescence Microscopy, Anal Chem 90(21) (2018) 12395-12403.

[5] K. Mallah, J. Quanico, D. Trede, F. Kobeissy, K. Zibara, M. Salzet, I. Fournier, Lipid Changes Associated with Traumatic Brain Injury Revealed by 3D MALDI-MSI, Anal Chem 90(17) (2018) 10568-10576.

[6] D.R.N. Vos, I. Jansen, M. Lucas, M.R.L. Paine, O.J. de Boer, S.L. Meijer, C.D. Savci-Heijink, H.A. Marquering, D.M. de Bruin, R.M.A. Heeren, S.R. Ellis, B. Balluff, Strategies for managing multipatient 3D mass spectrometry imaging data, J Proteomics 193 (2019) 184-191.

[7] M. Stoeckli, D. Staab, M. Staufenbiel, K.H. Wiederhold, L. Signor, Molecular imaging of amyloid beta peptides in mouse brain sections using mass spectrometry, Anal Biochem 311(1) (2002) 339.

[8] O. Philipson, A. Lord, M. Lalowski, R. Soliymani, M. Baumann, J. Thyberg, N. Bogdanovic, T. Olofsson, L.O. Tjernberg, M. Ingelsson, L. Lannfelt, H. Kalimo, L.N. Nilsson, The Arctic amyloidbeta precursor protein (AbetaPP) mutation results in distinct plaques and accumulation of $\mathrm{N}$ - and C-truncated Abeta, Neurobiol Aging 33(5) (2012) 1010 e1-13.

[9] N. Kakuda, T. Miyasaka, N. Iwasaki, T. Nirasawa, S. Wada-Kakuda, J. Takahashi-Fujigasaki, S. Murayama, Y. Ihara, M. Ikegawa, Distinct deposition of amyloid-beta species in brains with Alzheimer's disease pathology visualized with MALDI imaging mass spectrometry, Acta Neuropathol Commun 5(1) (2017) 73.

[10] P.M. Wehrli, W. Michno, K. Blennow, H. Zetterberg, J. Hanrieder, Chemometric Strategies for Sensitive Annotation and Validation of Anatomical Regions of Interest in Complex Imaging Mass Spectrometry Data, J Am Soc Mass Spectrom 30(11) (2019) 2278-2288.

[11] I. Kaya, W. Michno, D. Brinet, Y. lacone, G. Zanni, K. Blennow, H. Zetterberg, J. Hanrieder, Histology-Compatible MALDI Mass Spectrometry Based Imaging of Neuronal Lipids for Subsequent Immunofluorescent Staining, Anal Chem 89(8) (2017) 4685-4694. 
Enzlein et al. Computational Analysis of Amyloid Plaque Composition Submission to Anal. Chem.

[12] M. Schwartz, B. Meyer, B. Wirnitzer, C. Hopf, Standardized processing of MALDI imaging raw data for enhancement of weak analyte signals in mouse models of gastric cancer and Alzheimer's disease, Anal Bioanal Chem 407(8) (2015) 2255-64.

[13] W.M. Abdelmoula, M.S. Regan, B.G.C. Lopez, E.C. Randall, S. Lawler, A.C. Mladek, M.O. Nowicki, B.M. Marin, J.N. Agar, K.R. Swanson, T. Kapur, J.N. Sarkaria, W. Wells, N.Y.R. Agar, Automatic 3D Nonlinear Registration of Mass Spectrometry Imaging and Magnetic Resonance Imaging Data, Anal Chem 91(9) (2019) 6206-6216.

[14] T. Alexandrov, MALDI imaging mass spectrometry: statistical data analysis and current computational challenges, BMC Bioinformatics 13 Suppl 16 (2012) S11.

[15] Z. Niu, Z. Zhang, W. Zhao, J. Yang, Interactions between amyloid beta peptide and lipid membranes, Biochim Biophys Acta Biomembr (2018).

[16] I. Kaya, D. Brinet, W. Michno, S. Syvanen, D. Sehlin, H. Zetterberg, K. Blennow, J. Hanrieder, Delineating Amyloid Plaque Associated Neuronal Sphingolipids in Transgenic Alzheimer's Disease Mice (tgArcSwe) Using MALDI Imaging Mass Spectrometry, ACS Chem Neurosci 8(2) (2017) 347355.

[17] I. Kaya, E. Jennische, J. Dunevall, S. Lange, A.G. Ewing, P. Malmberg, A.T. Baykal, J.S. Fletcher, Spatial Lipidomics Reveals Region and Long Chain Base Specific Accumulations of Monosialogangliosides in Amyloid Plaques in Familial Alzheimer's Disease Mice (5xFAD) Brain, ACS Chem Neurosci 11(1) (2020) 14-24.

[18] L. Chavez-Gutierrez, L. Bammens, I. Benilova, A. Vandersteen, M. Benurwar, M. Borgers, S. Lismont, L. Zhou, S. Van Cleynenbreugel, H. Esselmann, J. Wiltfang, L. Serneels, E. Karran, H. Gijsen, J. Schymkowitz, F. Rousseau, K. Broersen, B. De Strooper, The mechanism of gammaSecretase dysfunction in familial Alzheimer disease, Embo J 31(10) (2012) 2261-74.

[19] C. Haass, C. Kaether, G. Thinakaran, S. Sisodia, Trafficking and proteolytic processing of APP, Cold Spring Harb Perspect Med 2(5) (2012) a006270.

[20] M.P. Kummer, M.T. Heneka, Truncated and modified amyloid-beta species, Alzheimers Res Ther 6(3) (2014) 28.

[21] M. Szaruga, S. Veugelen, M. Benurwar, S. Lismont, D. Sepulveda-Falla, A. Lleo, N.S. Ryan, T. Lashley, N.C. Fox, S. Murayama, H. Gijsen, B. De Strooper, L. Chavez-Gutierrez, Qualitative changes in human gamma-secretase underlie familial Alzheimer's disease, J Exp Med 212(12) (2015) 2003-13.

[22] M. Szaruga, B. Munteanu, S. Lismont, S. Veugelen, K. Horre, M. Mercken, T.C. Saido, N.S. Ryan, T. De Vos, S.N. Savvides, R. Gallardo, J. Schymkowitz, F. Rousseau, N.C. Fox, C. Hopf, B. De Strooper, L. Chavez-Gutierrez, Alzheimer's-Causing Mutations Shift Abeta Length by Destabilizing gamma-Secretase-Abetan Interactions, Cell 170(3) (2017) 443-456 e14.

[23] N. Matsumura, M. Takami, M. Okochi, S. Wada-Kakuda, H. Fujiwara, S. Tagami, S. Funamoto, Y. Ihara, M. Morishima-Kawashima, gamma-Secretase associated with lipid rafts: multiple interactive pathways in the stepwise processing of beta-carboxyl-terminal fragment, J Biol Chem 289(8) (2014) 5109-21. 
Enzlein et al. Computational Analysis of Amyloid Plaque Composition Submission to Anal. Chem.

[24] H. Chaudhary, S.W. Meister, H. Zetterberg, J. Lofblom, C. Lendel, Dissecting the Structural Organization of Multiprotein Amyloid Aggregates Using a Bottom-Up Approach, ACS Chem Neurosci 11(10) (2020) 1447-1457.

[25] W. Michno, I. Kaya, S. Nystrom, L. Guerard, K.P.R. Nilsson, P. Hammarstrom, K. Blennow, H. Zetterberg, J. Hanrieder, Multimodal Chemical Imaging of Amyloid Plaque Polymorphism Reveals Abeta Aggregation Dependent Anionic Lipid Accumulations and Metabolism, Anal Chem 90(13) (2018) 8130-8138.

[26] W. Michno, P. Wehrli, S.R. Meier, D. Sehlin, S. Syvanen, H. Zetterberg, K. Blennow, J. Hanrieder, Chemical imaging of evolving amyloid plaque pathology and associated Abeta peptide aggregation in a transgenic mouse model of Alzheimer's disease, J Neurochem (2019).

[27] I. Kaya, E. Jennische, S. Lange, A.T. Baykal, P. Malmberg, J.S. Fletcher, Brain Region Specific Amyloid Plaque Associated Myelin Lipid Loss, APOE Deposition and Disruption of the Myelin Sheath in Familial Alzheimer's Disease Mice, J Neurochem (2020).

[28] K. Erich, D.A. Sammour, A. Marx, C. Hopf, Scores for standardization of on-tissue digestion of formalin-fixed paraffin-embedded tissue in MALDI-MS imaging, Biochim Biophys Acta Proteins Proteom 1865(7) (2017) 907-915.

[29] T. Saito, Y. Matsuba, N. Mihira, J. Takano, P. Nilsson, S. Itohara, N. Iwata, T.C. Saido, Single App knock-in mouse models of Alzheimer's disease, Nat Neurosci 17(5) (2014) 661-3.

[30] R. Radde, T. Bolmont, S.A. Kaeser, J. Coomaraswamy, D. Lindau, L. Stoltze, M.E. Calhoun, F. Jaggi, H. Wolburg, S. Gengler, C. Haass, B. Ghetti, C. Czech, C. Holscher, P.M. Mathews, M. Jucker, Abeta42-driven cerebral amyloidosis in transgenic mice reveals early and robust pathology, EMBO Rep 7(9) (2006) 940-6.

[31] H. Sasaguri, P. Nilsson, S. Hashimoto, K. Nagata, T. Saito, B. De Strooper, J. Hardy, R. Vassar, B. Winblad, T.C. Saido, APP mouse models for Alzheimer's disease preclinical studies, Embo J 36(17) (2017) 2473-2487.

[32] J. Yang, R.M. Caprioli, Matrix sublimation/recrystallization for imaging proteins by mass spectrometry at high spatial resolution, Anal Chem 83(14) (2011) 5728-34.

[33] T. Schramm, Z. Hester, I. Klinkert, J.P. Both, R.M.A. Heeren, A. Brunelle, O. Laprevote, N. Desbenoit, M.F. Robbe, M. Stoeckli, B. Spengler, A. Rompp, imzML--a common data format for the flexible exchange and processing of mass spectrometry imaging data, J Proteomics 75(16) (2012) 5106-5110.

[34] S. Gibb, K. Strimmer, MALDlquant: a versatile R package for the analysis of mass spectrometry data, Bioinformatics 28(17) (2012) 2270-1.

[35] A. Rosenfeld, J.L. Pfaltz, Sequential Operations in Digital Picture Processing., J. ACM 13(4) (1966) 471-494.

[36] R.M. Haralick, L.G. Shapiro, Computer and Robot Vision (1st. ed.), Addison-Wesley Longman Publishing Co., Inc., USA.1992. 
Enzlein et al. Computational Analysis of Amyloid Plaque Composition Submission to Anal. Chem.

[37] H.C. Diehl, B. Beine, J. Elm, D. Trede, M. Ahrens, M. Eisenacher, K. Marcus, H.E. Meyer, C. Henkel, The challenge of on-tissue digestion for MALDI MSI- a comparison of different protocols to improve imaging experiments, Anal Bioanal Chem 407(8) (2015) 2223-43.

[38] A. Buck, B. Heijs, B. Beine, J. Schepers, A. Cassese, R.M.A. Heeren, L.A. McDonnell, C. Henkel, A. Walch, B. Balluff, Round robin study of formalin-fixed paraffin-embedded tissues in mass spectrometry imaging, Anal Bioanal Chem 410(23) (2018) 5969-5980.

[39] A. Ly, R. Longuespee, R. Casadonte, P. Wandernoth, K. Schwamborn, C. Bollwein, C. Marsching, K. Kriegsmann, C. Hopf, W. Weichert, J. Kriegsmann, P. Schirmacher, M. Kriegsmann, S.O. Deininger, Site-to-Site Reproducibility and Spatial Resolution in MALDI-MSI of Peptides from Formalin-Fixed Paraffin-Embedded Samples, Proteomics Clin Appl 13(1) (2019) e1800029.

[40] M. Citron, T. Oltersdorf, C. Haass, L. McConlogue, A.Y. Hung, P. Seubert, C. Vigo-Pelfrey, I. Lieberburg, D.J. Selkoe, Mutation of the beta-amyloid precursor protein in familial Alzheimer's disease increases beta-protein production, Nature 360(6405) (1992) 672-4.

[41] X.D. Cai, T.E. Golde, S.G. Younkin, Release of excess amyloid beta protein from a mutant amyloid beta protein precursor, Science 259(5094) (1993) 514-6.

[42] S.F. Lichtenthaler, R. Wang, H. Grimm, S.N. Uljon, C.L. Masters, K. Beyreuther, Mechanism of the cleavage specificity of Alzheimer's disease gamma-secretase identified by phenylalaninescanning mutagenesis of the transmembrane domain of the amyloid precursor protein, Proc Natl Acad Sci U S A 96(6) (1999) 3053-8.

[43] C. Nilsberth, A. Westlind-Danielsson, C.B. Eckman, M.M. Condron, K. Axelman, C. Forsell, C. Stenh, J. Luthman, D.B. Teplow, S.G. Younkin, J. Naslund, L. Lannfelt, The 'Arctic' APP mutation (E693G) causes Alzheimer's disease by enhanced Abeta protofibril formation, Nat Neurosci 4(9) (2001) 887-93.

[44] D. Kwart, A. Gregg, C. Scheckel, E.A. Murphy, D. Paquet, M. Duffield, J. Fak, O. Olsen, R.B. Darnell, M. Tessier-Lavigne, A Large Panel of Isogenic APP and PSEN1 Mutant Human iPSC Neurons Reveals Shared Endosomal Abnormalities Mediated by APP beta-CTFs, Not Abeta, Neuron 104(2) (2019) 256-270 e5.

[45] M. Holzlechner, K. Strasser, E. Zareva, L. Steinhauser, H. Birnleitner, A. Beer, M. Bergmann, R. Oehler, M. Marchetti-Deschmann, In Situ Characterization of Tissue-Resident Immune Cells by MALDI Mass Spectrometry Imaging, J Proteome Res 16(1) (2017) 65-76.

[46] L. Ertöz, M. Steinbach, V. Kumar, Finding Clusters of Different Sizes, Shapes, and Densities in Noisy, High Dimensional Data, Proceedings of the Third SIAM International Conference on Data Mining, San Francisco, CA, USA, 2003.

[47] N. Otsu, A Threshold Selection Method from Gray-Level Histograms. , IEEE Transactions on Systems, Man and Cybernetics. 9(1) (1979) 62-66.

[48] N. Coudray, J.L. Buessler, J.P. Urban, Robust threshold estimation for images with unimodal histograms, Pattern Recognition Letters 31(9) (2010) 1010-1019. 
Enzlein et al. Computational Analysis of Amyloid Plaque Composition Submission to Anal. Chem.

[49] B.R. Pinzer, M. Cacquevel, P. Modregger, S.A. McDonald, J.C. Bensadoun, T. Thuering, P. Aebischer, M. Stampanoni, Imaging brain amyloid deposition using grating-based differential phase contrast tomography, Neuroimage 61(4) (2012) 1336-46.

[50] M. Querol-Vilaseca, M. Colom-Cadena, J. Pegueroles, R. Nunez-Llaves, J. Luque-Cabecerans, L. Munoz-Llahuna, J. Andilla, O. Belbin, T.L. Spires-Jones, E. Gelpi, J. Clarimon, P. Loza-Alvarez, J. Fortea, A. Lleo, Nanoscale structure of amyloid-beta plaques in Alzheimer's disease, Sci Rep 9(1) (2019) 5181.

[51] T. Liebmann, N. Renier, K. Bettayeb, P. Greengard, M. Tessier-Lavigne, M. Flajolet, ThreeDimensional Study of Alzheimer's Disease Hallmarks Using the iDISCO Clearing Method, Cell Rep 16(4) (2016) 1138-1152.

[52] T. Iwatsubo, A. Odaka, N. Suzuki, H. Mizusawa, N. Nukina, Y. Ihara, Visualization of A beta 42(43) and $A$ beta 40 in senile plaques with end-specific $A$ beta monoclonals: evidence that an initially deposited species is A beta 42(43), Neuron 13(1) (1994) 45-53.

[53] S.A. Gravina, L. Ho, C.B. Eckman, K.E. Long, L. Otvos, Jr., L.H. Younkin, N. Suzuki, S.G. Younkin, Amyloid beta protein (A beta) in Alzheimer's disease brain. Biochemical and immunocytochemical analysis with antibodies specific for forms ending at A beta 40 or A beta 42(43), J Biol Chem 270(13) (1995) 7013-6.

[54] W. Michno, S. Nystrom, P. Wehrli, T. Lashley, G. Brinkmalm, L. Guerard, S. Syvanen, D. Sehlin, I. Kaya, D. Brinet, K.P.R. Nilsson, P. Hammarstrom, K. Blennow, H. Zetterberg, J. Hanrieder, Pyroglutamation of amyloid-betax-42 (Abetax-42) followed by Abeta1-40 deposition underlies plaque polymorphism in progressing Alzheimer's disease pathology, J Biol Chem 294(17) (2019) 6719-6732.

[55] W. Michno, P.M. Wehrli, K. Blennow, H. Zetterberg, J. Hanrieder, Molecular imaging mass spectrometry for probing protein dynamics in neurodegenerative disease pathology, J Neurochem 151(4) (2019) 488-506.

[56] C. Guardia-Laguarta, M. Pera, J. Clarimon, J.L. Molinuevo, R. Sanchez-Valle, A. Llado, M. Coma, T. Gomez-Isla, R. Blesa, I. Ferrer, A. Lleo, Clinical, neuropathologic, and biochemical profile of the amyloid precursor protein I716F mutation, J Neuropathol Exp Neurol 69(1) (2010) 53-9. 